\title{
A Poincaré Lemma for Whitney-de Rham complex
}

\author{
By
}

\author{
Hou-Yi Chen
}

\begin{abstract}
Let $M$ be a real analytic manifold, $Z$ a closed subanalytic subset of $M$. We show that the Whitney-de Rham complex over $Z$ is quasi-isomorphic to the constant sheaf $\mathbb{C}_{Z}$.
\end{abstract}

\section{Contents}

1 Introduction 1

2 Review on Whitney and formal cohomology functors 2

3 Main result 3

\section{Introduction}

In [KS96], Kashiwara-Schapira introduced the Whitney functor (real case) and formal cohomology functor (complex case), then they introduced the notion of ind-sheaves and they also defined Grothendieck six operations in this framework in [KS01]. As applications, they defined the Whitney $\mathcal{C}^{\infty}$ functions and Whitney holomorphic functions on the subanalytic site as examples of ind-sheaves. The more elementary study for sheaves on the subanalytic site is performed in [Pr08] and $[\operatorname{Pr} 12]$.

Let $M$ be a real analytic manifold, by Poincaré Lemma, it is well-known that the de Rham complex over $M$ is isomorphic to $\mathbb{C}_{M}$. The aim of this paper is to show that a theorem of [BP08] follows easily from a deep result of Kashiwara on regular holonomic $\mathscr{D}$-module [K84] and the Whitney functor of [KS96]. More precisely, we show that

Main theorem(=Theorem 3.3.) Let $M$ be a real analytic manifold of dimension $n$ and $Z$ a closed subanalytic subset of $M$. Then we have:

$$
\mathbb{C}_{Z} \stackrel{\sim}{\longrightarrow}\left(0 \longrightarrow \mathcal{W}_{M, Z}^{\infty} \stackrel{d}{\longrightarrow} \mathcal{W}_{M, Z}^{(\infty, 1)} \stackrel{d}{\longrightarrow} \cdots \stackrel{d}{\longrightarrow} \mathcal{W}_{M, Z}^{(\infty, n)} \longrightarrow 0\right)
$$


where $\mathcal{W}_{M, Z}^{\infty}$ denotes the sheaf of Whitney functions on $Z$ and $\mathcal{W}_{M, Z}^{(\infty, i)}$ denotes the sheaf of differential forms of degree $i$ with coefficients in $\mathcal{W}_{M, Z}^{\infty}$ for each $i$, i.e., the Whitney-de Rham complex is isomorhpic to $\mathbb{C}_{Z}$.

Acknowledgments. I would like to thank Pierre Schapira for suggesting this problem to me and for many useful conversations.

\section{Review on Whitney and formal cohomology functors}

In this section, we review some results on Whitney and formal cohomology functors. References are made to [KS96], [KS01], [Pr08], [Pr12] and [S12].

Let $M$ be a real analytic manifold, we denote by $\mathcal{A}_{M}, \mathcal{C}_{M}^{\infty}$ the sheaf of complex-valued real analytic functions, $\mathcal{C}^{\infty}$-functions on $M$. We denote by $\mathcal{D}_{M}$ the sheaf of rings on $M$ of finite-order differential operators with coefficients in $\mathcal{A}_{M}$.

We denote by $\operatorname{Mod}_{\mathbb{R}-c}\left(\mathbb{C}_{M}\right)$ the abelian category of $\mathbb{R}$-constructible sheaves on $M$ and $\operatorname{Mod}\left(\mathcal{D}_{M}\right)$ the abelian category of left $\mathcal{D}_{M}$-modules. We also denote by $\mathrm{D}_{\mathbb{R}-\mathrm{c}}^{\mathrm{b}}\left(\mathbb{C}_{M}\right)$ the bounded derived category consisting of objects whose cohomology groups belong to $\operatorname{Mod}_{\mathbb{R}-\mathrm{c}}\left(\mathbb{C}_{M}\right)$ and $\mathrm{D}^{\mathrm{b}}\left(\mathcal{D}_{M}\right)$ the derived category of $\operatorname{Mod}\left(\mathcal{D}_{M}\right)$ with bounded cohomologies.

Definition 2.1. Let $Z$ be a closed subset of $M$. We denote by $\mathcal{I}_{M, Z}^{\infty}$ the sheaf of $\mathcal{C}^{\infty}$ functions on $M$ vanishing up to infinite order on $Z$.

Definition 2.2. A Whitney function on a closed subset $Z$ of $M$ is an indexed family $F=\left(F^{k}\right)_{k \in \mathbb{N}}$ consisting of continuous functions on $Z$ such that $\forall m \in \mathbb{N}$, $\forall k \in \mathbb{N}^{n},|k| \leq m, \forall x \in Z, \forall \varepsilon>0$ there exists a neighborhood such that $\forall y, z \in U \cap Z$

$$
\left|F^{k}(z)-\sum_{|j+k| \leq m} \frac{(z-y)^{j}}{j !} F^{j+k}(y)\right| \leq \varepsilon d(y, z)^{m-|k|} .
$$

We denote by $W_{M, Z}^{\infty}$ the space of Whitney $C^{\infty}$ functions on $Z$. We denote by $\mathcal{W}_{M, Z}^{\infty}$ the sheaf $U \stackrel{\mapsto}{ } \mapsto W_{U, U \cap Z}^{\infty}$.

In [KS96], the authors defined the Whitney tensor product functor as follows:

$$
\cdot \stackrel{\mathrm{w}}{\otimes} \mathcal{C}_{M}^{\infty}: \operatorname{Mod}_{\mathbb{R}-\mathrm{c}}\left(\mathbb{C}_{M}\right) \rightarrow \operatorname{Mod}\left(\mathcal{D}_{M}\right)
$$

in the following way: let $U$ be an open subanalytic subset of $M$ and $Z=$ $M \backslash U$. Then $\mathbb{C}_{U} \stackrel{\mathrm{w}}{\otimes} \mathcal{C}_{M}^{\infty}=\mathcal{I}_{M, Z}^{\infty}$ and $\mathbb{C}_{Z} \stackrel{\mathrm{w}}{\otimes} \mathcal{C}_{M}^{\infty}=\mathcal{W}_{M, Z}^{\infty}$. This functor is exact and extends as a functor in the derived category, from $\mathrm{D}_{\mathbb{R}-\mathrm{c}}^{\mathrm{b}}\left(\mathbb{C}_{M}\right)$ to $\mathrm{D}^{\mathrm{b}}\left(\mathcal{D}_{M}\right)$. Moreover the sheaf $F \stackrel{\mathrm{w}}{\otimes} \mathcal{C}_{M}^{\infty}$ is soft for any $\mathbb{R}$-constructible sheaf $F$.

Now let $X$ be a complex manifold and we denote by $\mathscr{D}_{X}$ the sheaf of rings on $X$ of finite-order differential operators. We still denote by $X$ the real underlying manifold and we denote by $\bar{X}$ the complex manifold conjugate to $X$. One defines the functor of formal cohomology as follows:

Let $F \in \mathrm{D}_{\mathbb{R}-\mathrm{c}}^{\mathrm{b}}\left(\mathbb{C}_{X}\right)$, we set 


$$
F \stackrel{\mathrm{w}}{\otimes} \mathscr{O}_{X}=\operatorname{RH}_{\mathcal{H}} m_{\mathscr{D}_{\bar{X}}}\left(\mathscr{O}_{\bar{X}}, F \stackrel{\mathrm{w}}{\otimes} \mathcal{C}_{X}^{\infty}\right),
$$

where $\mathscr{D}_{\bar{X}}$ denotes the sheaf of rings on $\bar{X}$ of finite-order differential operators.

Let $M$ be a real analytic manifold, $X$ a complexification of $M, \imath: M \hookrightarrow X$ the embedding. We recall the following result.

Theorem 2.3. ([KS96] Theorem 5.10.) Let $F \in \mathrm{D}_{\mathbb{R}-c}^{\mathrm{b}}\left(\mathbb{C}_{M}\right)$. Then we have

$$
\imath_{*} F \stackrel{\mathrm{w}}{\otimes} \mathscr{O}_{X} \simeq \imath_{*}\left(F \stackrel{\mathrm{w}}{\otimes} \mathcal{C}_{M}^{\infty}\right) .
$$

In particular,

$$
\mathbb{C}_{M} \stackrel{\mathrm{w}}{\otimes} \mathcal{O}_{X} \simeq \mathcal{C}_{M}^{\infty}
$$

The following proposition is the key point of this note.

Proposition 2.4. ([KS96] Corollary 6.2) Let $\mathfrak{M}$ be a regular holonomic $\mathscr{D}_{X}$ module, and let $F$ be an object of $\mathrm{D}_{\mathbb{R}-c}^{\mathrm{b}}\left(\mathbb{C}_{X}\right)$. Then, the natural morphism:

$$
R \mathcal{H} \operatorname{Hom}_{\mathscr{D}_{X}}\left(\mathfrak{M}, F \otimes \mathscr{O}_{X}\right) \rightarrow R \mathcal{H o m} \mathscr{D}_{X}\left(\mathfrak{M}, F \stackrel{\mathrm{w}}{\otimes} \mathscr{O}_{X}\right) .
$$

is an isomorphism.

\section{Main result}

Let $X$ be a complex manifold of dimension $n$. We denote by $\mathscr{D}_{X}$ the sheaf of rings of finite-order differential operators and $\Theta_{X}$ the sheaf of vector fields on $X$.

First we recall the following basic result in $\mathscr{D}_{X}$-module theory.

Proposition 3.1. ([K03] Proposition 1.6) The complex

$0 \rightarrow \mathscr{D}_{X} \otimes_{\mathscr{O}_{X}} \bigwedge^{n} \Theta_{X} \rightarrow \cdots \rightarrow \mathscr{D}_{X} \otimes_{\mathscr{O}_{X}} \bigwedge^{2} \Theta_{X} \rightarrow \mathscr{D}_{X} \otimes_{\mathscr{O}_{X}} \Theta_{X} \rightarrow \mathscr{D}_{X} \rightarrow \mathscr{O}_{X} \rightarrow 0$ is exact.

Lemma 3.2. Let $\mathscr{M}$ be a left $\mathscr{D}_{X}$-module. Then we have

$$
R \mathcal{H} \operatorname{Hom}_{\mathscr{D}_{X}}\left(\mathscr{O}_{X}, \mathscr{M}\right) \simeq\left[\mathscr{M} \rightarrow \Omega_{X}^{1} \otimes_{\mathscr{O}_{X}} \mathscr{M} \rightarrow \cdots \rightarrow \bigwedge^{n} \Omega_{X}^{1} \otimes_{\mathscr{O}_{X}} \mathscr{M}\right] .
$$

Proof. By Proposition 3.1, we have

$$
\begin{aligned}
& R \mathcal{H o m} \mathscr{D}_{X}\left(\mathscr{O}_{X}, \mathscr{M}\right) \\
\simeq & {\left[\mathscr{M} \rightarrow \mathcal{H o m}_{\mathscr{D}_{X}}\left(\mathscr{D}_{X} \otimes_{\mathscr{O}_{X}} \Theta_{X}, \mathscr{M}\right) \rightarrow \cdots \rightarrow \mathcal{H} m_{\mathscr{D}_{X}}\left(\mathscr{D}_{X} \otimes_{\mathscr{O}_{X}} \bigwedge^{n} \Theta_{X}, \mathscr{M}\right)\right] } \\
\simeq & {\left[\mathscr{M} \rightarrow \mathcal{H o m}_{\mathscr{O}_{X}}\left(\Theta_{X}, \mathscr{M}\right) \rightarrow \cdots \rightarrow \mathcal{H o m} \mathscr{O}_{X}\left(\bigwedge^{n} \Theta_{X}, \mathscr{M}\right)\right] } \\
\simeq & {\left[\mathscr{M} \rightarrow \Omega_{X}^{1} \otimes_{\mathscr{O}_{X}} \mathscr{M} \rightarrow \cdots \rightarrow \bigwedge^{n} \Omega_{X}^{1} \otimes_{\mathscr{O}_{X}} \mathscr{M}\right] }
\end{aligned}
$$


where $\Omega_{X}^{1}:=\mathcal{H o m}_{\mathscr{O}_{X}}\left(\Theta_{X}, \mathscr{O}_{X}\right)$.

Let $M$ be a real analytic manifold, $X$ a complexification of $M$ and $Z$ a closed subanalytic subset of $M$. We denote by $\Omega_{X}^{1}$ the sheaf of differential one-form on $X$ and

(3.1) $\mathscr{A}_{Z}^{(i)}:=\bigwedge^{i} \Omega_{X}^{1} \otimes_{\mathscr{O}_{X}}\left(\mathbb{C}_{Z} \otimes \mathscr{O}_{X}\right)$,

(3.2) $\mathcal{W}_{M, Z}^{(\infty, i)}:=\bigwedge^{i} \Omega_{X}^{1} \otimes_{\mathscr{O}_{X}}\left(\mathbb{C}_{Z} \stackrel{\mathrm{w}}{\otimes} \mathscr{O}_{X}\right) \simeq \bigwedge^{i} \Omega_{X}^{1} \otimes_{\mathscr{O}_{X}}\left(\mathbb{C}_{Z} \stackrel{\mathrm{w}}{\otimes} \mathcal{C}_{M}^{\infty}\right)$.

Now we are ready to prove the main theorem of this note below.

Theorem 3.3. $\quad$ Let $M$ be a real analytic manifold of dimension $n$ and $Z$ a closed subanalytic subset of $M$. Then we have:

$$
\mathbb{C}_{Z} \stackrel{\sim}{\longrightarrow}\left(0 \longrightarrow \mathcal{W}_{M, Z}^{\infty} \stackrel{d}{\longrightarrow} \mathcal{W}_{M, Z}^{(\infty, 1)} \stackrel{d}{\longrightarrow} \cdots \stackrel{d}{\longrightarrow} \mathcal{W}_{M, Z}^{(\infty, n)} \longrightarrow 0\right)
$$

where $\mathcal{W}_{M, Z}^{\infty}$ denotes the sheaf of Whitney functions on $Z$ and $\mathcal{W}_{M, Z}^{(\infty, i)}$ denotes the sheaf of differential forms of degree $i$ with coefficients in $\mathcal{W}_{M, Z}^{\infty}$ for each $i$ which are defined in (3.2), i.e., the Whitney-de Rham complex is isomorhpic to $\mathbb{C}_{Z}$.

Proof. Take $\mathfrak{M}=\mathscr{O}_{X}$ and $F=\mathbb{C}_{Z}$ in Proposition 2.4.

On the one hand, we show that the left hand side of (2.1) is $\mathbb{C}_{Z}$. By Theorem 2.3 and Lemma 3.2 , we get the following complex

$$
0 \longrightarrow \mathbb{C}_{M} \longrightarrow \mathscr{A}_{M}^{(0)} \stackrel{d}{\longrightarrow} \cdots \stackrel{d}{\longrightarrow} \mathscr{A}_{M}^{(n)} \longrightarrow 0
$$

which is exact by Poincaré lemma where $\mathscr{A}_{M}^{(i)}$ 's are defined in (3.1) by taking $Z=M$. Tensoring $\mathbb{C}_{Z}$, we obtain the following exact sequence

$$
0 \longrightarrow \mathbb{C}_{Z} \longrightarrow \mathscr{A}_{Z}^{(0)} \stackrel{d}{\longrightarrow} \cdots \stackrel{d}{\longrightarrow} \mathscr{A}_{Z}^{(n)} \longrightarrow 0 .
$$

Therefore,

$$
\mathbb{C}_{Z} \stackrel{\sim}{\longrightarrow}\left(0 \longrightarrow \mathscr{A}_{Z}^{(0)} \stackrel{d}{\longrightarrow} \cdots \stackrel{d}{\longrightarrow} \mathscr{A}_{Z}^{(n)} \longrightarrow 0\right) .
$$

On the other hand, the right hand side of (2.1) is the Whitney-de Rham complex

$$
0 \longrightarrow \mathcal{W}_{M, Z}^{\infty} \stackrel{d}{\longrightarrow} \mathcal{W}_{M, Z}^{(\infty, 1)} \stackrel{d}{\longrightarrow} \cdots \stackrel{d}{\longrightarrow} \mathcal{W}_{M, Z}^{(\infty, n)} \longrightarrow 0 .
$$

Now the result follows from the isomoprhism of (2.1).

\section{References}

[BP08] J-P Brasselet and M. J. Pflaum, On the homology of algebras of Whitney functions over subanalytic sets, Annals of Mathematics, 167 (2008), 152 .

[K84] M. Kashiwara, The Riemann-Hilbert problem for holonomic systems, Publ. Res. Inst. Math. Sci. 20 p. 319-365, (1984). 
[K03] — D-modules and microlocal calculus, Translations of Mathematical Monographs, Vol. 217, Amer. Math. Soc., 2003.

[KS96] M. Kashiwara and P. Schapira, Moderate and formal cohomology associated with constructible sheaves, Mémoires Soc. Math. France 64, (1996).

[KS01] — Ind-sheaves, Astérisque 271 (2001).

[Pr08] L. Prelli, Sheaves on subanalytic sites, Rendiconti del Seminario Matematico dell'Universit di Padova, 120 p. 167-216 (2008).

[Pr12] — Microlocalization of subanalytic sheaves, Mém. Soc. Math. France (2012).

[S12] P. Schapira, Three lectures on Algebraic Microlocal Analysis, Northwestern University May 2012, arXiv:1206.1435.

Institute of Mathematics, Academia Sinica, Taipei 106, Taiwan

E-mail address: houyi@math.sinica.edu.tw 